\title{
Discriminación de precios y competencia imperfecta en los mercados electrónicos
}

\author{
Andrade Castro, Jesús Alberto * \\ Córdova, Evelyn**
}

\section{Resumen}

El uso de las nuevas tecnologías de información en el desarrollo del comercio electrónico genera diferencias en los niveles de información que poseen los agentes del mercado; ello posibilita la formación de precios discriminados y por ende el desarrollo de mercados de competencia imperfecta, desplazando al modelo clásico de competencia perfecta como paradigma para el entendimiento de la formación de precios. Esta investigación analiza cómo se forman los precios de los bienes que se comercializan en los nuevos mercados electrónicos, bajo la perspectiva de los estudios previos de Pigou, quién analizó este aspecto basado en la satisfacción máxima de las necesidades del consumidor y su deseo por obtener el bien. Los resultados muestran diferencias entre lo que se entiende por discriminación de precios y diferenciación de precios. Se concluye que los niveles de información del comercio electrónico generan asimetrias basadas en una sobre abundancia de información y, que tales diferencias, fundamentan lo inapropiado del modelo clásico de competencia perfecta para entender los mecanismos de formación de precios en los mercados electrónicos.

Palabras clave: Discriminación de precios, formación de precios, comercio electrónico, mercado electrónico, modelo de competencia imperfecta.

\section{Recibido: 01-01-30. Aceptado: 01-04-04}

* Economista. Magister en Computación Aplicada y Master in Management Information Systems. Coordinador del programa de Maestria en Planíficación y Gerencia de Ciencia y Tecnología de LUZ. Profesor Titular del Departamento de Matemáticas y Computación. Facultad de Ciencias. Universidad del Zulia. Maracaibo, Venezuela. email address: Jandrade luz.ve

** Economista. Candidata a Magister Scientiarum en Planificación y Gerencia en Ciencia y Tecnología LUZ-

1 Esta investigación fue financiada con fondos del Consejo de Desarrollo Científico y Humanístico (CONDES) de La Universidad del Zulia. LUZ (No. 1386-99). 


\section{Price Discrimination and Imperfect Competition in Electronic Markets}

\section{Abstract}

The use of new informational technologies in the development of electronic commerce generates differences in the levels of information among market agents: and this makes possible the formation of discriminatory prices and the development of imperfect competitive markets, replacing the classic model of perfect competition as the paradigm for understanding price formation. This paper analyzes how the prices of electronic goods are formulated in these new markets through the perspective of previous studies by Pigou, who analyzed this aspect based on maximum satisfaction of consumer needs and their desire to obtain the goods. The results show differences between what is understood as price discrimination and price differentiation. The conclusion is that levels of information in electronic commerce generate asymmetries in an excessive abundance of information, and that these differences cause the basic problem in making the classic model of perfect competition inadequate in the analysis of mechanisms of price formation in electronic markets.

Key words: Price discrimination, price formation, electronic commerce, electronic market, imperfect competition model.

\section{Introducción}

Se ha argumentado con cierta intensidad, que el comercio electrónico estấ libre de todo tipo de restricciones de carácter geográfico y que por lo tanto existe una tendencia a disminuir los precios de los productos que se ofrecen en las redes de datos; ello bajo el supuesto de que este tipo de comercio elimina los costos en los cuales se incurre en los tradicionales comercios físicos. Costos asociados principalmente con el transporte, fletes y seguros que se requieren para que los bienes lleguen finalmente al consumidor parecen ser los primeros en reducirse y en ocasiones eliminarse. La lógica hace pensar que la eliminación de ciertos costos de producción y transporte de los bienes permitirá disminuir los precios que el consumidor final tendrá que pagar por adquirirlos. Sin embargo, exis- ten otros tipos de costos que no se reflejan directamente en su producción, sino que están relacionados por el tipo de mercados donde se comercializan; particularmente, aquellos costos asociados a la búsqueda de información que forman parte del desarrollo de los modelos de competencia de mercados.

No se puede negar que los mercados electrónicos, tan en auge hoy día, se caracterizan por una abundancia de información incentivada por las modernas tecnologías de información. Los productos que se comercializan en esos mercados electrónicos poseen ese componente extra de información que permite desarrollar otros tipos de mecanismos a la hora de fijar sus precios, distintos al de los costos de producción.

Aunque es difícil determinar si el éxito del comercio electrónico está asociado al dinamismo de la economía mun- 
dial en tiempos de globalización, es de suponer que el desarrollo de la internet $y$ de la World Wide Web -WWW- ha tenido un significativo impacto en la forma como se ha desarrollado el comercio en los últimos tiempos. Aún antes de que la explosión tecnológica apareciera -para llevar a cabo el comercio electrónico-, los mercados han estado sujetos a desviaciones en el nivel de información disponible a los agentes que en él participan.

Los análisis económicos siempre suponen que el proveedor es el mismo productor del bien y que el consumidor es el agente de la economía que en última instancia demanda el bien. Con el comercio electrónico el detallista actúa como el mismo productor $y$, el consumidor puede ser un agente intermedio de la economia; así que en el análisis de la formación de precios de bienes, los detallistas -tanto consumidores como productores- cumplen un rol más directo en el intercambio. En el comercio electrónico, un productor puede proveer directamente al consumidor final con los bienes y servicios que él suministra y, el consumidor final, puede contactar directamente al productor o al proveedor del bien que él necesita para satisfacer sus necesidades.

Los compradores buscan información acerca de proveedores de bienes y servicios, bienes y servicios en si mismos $y$, precios, términos y condiciones para llevar a cabo las transacciones. Por su parte, el vendedor deseará conocer el pa- trón de consumo de un determinado tipo de consumidor que pudiera interesarse por sus productos, el número de potenciales consumidores y el estado en general de precios de los productos y servicios que él ofrece. Sin embargo, el cúmulo de información que cada uno de esos agentes puede obtener y ofrecer no es de la misma calidad, ni del mismo nivel para cada uno de ellos, puesto que el mercado en sí mismo es asimétrico con respecto a la actuación de cada agente $y$, esas asimetrías, suponen diferencias en el nivel de información.

La realidad señala que el comercio electrónico puede ser complementario del comercio tradicional y por lo tanto el tipo de competencia que genera no excluye la posibilidad de entrar en competencia con los bienes y servicios que se comercializan en los mercados físicos.

Sarkar et al (1995) afirman que el comercio electrónico conducirá a una desintermediación ${ }^{2}$ de la actividad económica puesto que los consumidores en linea inter actuarán directamente con los proveedores en línea y por lo tanto los precios disminuirán y ello es debido a la desaparición de los márgenes que se deben calcular para los intermediarios. Por su parte, Schmitz (2000) afirma que los intermediarios proveen un número amplio de servicios que son necesarios para el comercio y que su participación no está bajo amenaza por la difusión del comercio electrónico, entre otros servicios se

2 Desplazamiento o eliminación de los intermediarios del mercado, al capacitar las transacciones directas con los compradores y consumidores sin participación de otros agentes (Wigand, 1997, p. 4). 
encuentran los siguientes: sostener inventarios para poder dar inmediato reparto, reducción de asimetrías de información a través de la reputación, recolectar, organizar y evaluar información dispersa en la sociedad.

La ventaja de la información estará del lado de quien posea los procedimientos y mecanismos más eficientes al momento de establecer una negociación, en tal sentido los avances de las tecnologías de información y de comunicación en el apoyo del comercio electrónico generan mayores y beneficiosas asimetrias de información para quienes posean tales tecnologias, puesto que les permite desarrollar procesos inteligentes en detrimento de quienes sólo hacen uso de las tecnologías en forma pasiva.

La gran diferencia que existe, en términos de las asimetrías de información que se producen en el comercio físico tradicional, comparada con las ocurridas con el comercio electrónico, está dada en que los niveles de información del comercio físico son consecuencia de una escasez de información y los diversos niveles de información que generan las asimetrias en el comercio electrónico están basados en una sobre abundancia de información impulsada por las tecnologías de información.

Hasta principios del siglo $X X_{i}$ el modelo imperante para explicar el comportamiento de los productores y consumidores en el mercado - $y$ al mercado mismo -, era el Modelo de Competencia Perfecta. Sin embargo, en el modelo neo-clásico de mercado las desviaciones en el nivel de información no deberian ocurrir; por lo tanto, para entender el mecanismo de formación de precios en los mercados electrónicos se debe partir de modelos más realistas de competencia y en tal sentido lo realista se refiere a mercados de competencia imperfecta.

A pesar del aparente dominio del modelo de competencia perfecta para explicar todo lo concerniente al mercado y su funcionamiento, algunos economistas del siglo $X X$ estudiaron las discrepancias que se observaban entre la realidad y los postulados teóricos que sustentan al modelo. Las criticas que hicieron Hotelling (1929), Pigou (1946) y Robinson (1934) al Modelo de Competencia Perfecta, contribuyeron a crear nuevos modelos téricos que explican, en forma fidedigna, lo que realmente sucede en los mercados. No sólo estos modelos se ajustaban a los mercados del siglo $X X$; si no que aún hoy, muchas de las estructuras de la organización industrial son explicadas por los postulados teóricos denominados como modelos de Competencia Imperfecta: Competencia Monopolística, Monopolio (Simple y Discriminado) y Oligopolio (Puro y Diferencial).

Todos los modelos señalados con anterioridad, de alguna manera suponen que el mecanismo de formación de precios de los bienes está condicionado por el nivel de información que posee cada uno de los agentes del mercado. Y es que la forma como se distribuye la información en el mercado, no es simétrica entre todos los participantes, debido a que tales agentes pueden esconder, crear, distorsionar o proveer información para beneficio propio. Particularmente, Akerlof (1970) describió la influencia de los diferentes grados de calidad de los producios en relación a las incertidumbres de los compradores con insuficiente informa- 
ción. Baja calidad de los productos son más baratos de producir, aunque pueden ser menos deseables para el consumo. Cuando un potencial comprador no tiene suficiente información acerca de la calidad del producto, este tiende a desear pagar el precio de calidad más bajo; este análisis de Akerlof corresponde a las desviaciones de información en términos de la calidad del producto.

Este trabajo analiza las diferencias en los niveles de información que poseen los agentes del mercado, que posibilitan la formación de precios discriminados y por ende el desarrollo de mercados de competencia imperfecta, desplazando al modelo clásico de competencia perfecta como paradigma para el entendimiento de la formación de precios.

\section{La emancipación ${ }^{3}$ del mito de la competencia perfecta: Poder monopolístico y discriminación de precios}

El detonante que permitió la caída del mito de la Competencia Perfecta como modelo para explicar el comportamiento del mercado y sus agentes, se resume en dos palabras: poder monopolístico. El poder monopolístico no sólo se refiere al poder 0 injerencia que tiene el vendedor de fijar el precio del bien, más allá del costo marginal de producción; también se refiere al poder que tiene el vendedor Pigou (1946) lo identificó como monopolista para diferenciar su producto en el mercado y captar más consumidores a través de atractivos (artificiales 0 reales) que, sumados a las características propias del producto, le permite poder justificar el cobro de un precio que no es igual al costo marginal de producirlo.

El poder monopolístico del vendedor rompe con dos de los fundamentos del Modelo de Competencia Perfecta: primero, el vendedor monopolista no es precio aceptante; puesto que tiene suficiente poder en el mercado para fijar el precio, mientras que al consumidor tan sólo le corresponde aceptar el precio ${ }^{4}$; segundo, el producto no es homogéneo; el vendedor monopolista puede modificar el producto y crear diferencias reales (alteraciones físico-químicas) 0 artificiales (publicidad, crear versiones, empaquetarlos, degradarlos, condicionarlo al número de veces que se usan, etc) para manipular al consumidor.

Cualquier mercado imperfecto, a excepción del de monopolio simple y el de oligopolio puro -donde se fija un único precio-, está en capacidad de manejar al mismo tiempo diferentes precios para un

3 Palabra utilizada por Robinson (1934) para referirse a la caida del Modelo de Competencia Pertecta.

4 Pigou en "La Economia del Bienestar" (1946), afirma que: "La pérdida de un cliente comprador le importa menos al vendedor monopolista que a cualquiera de los compradores monopolistas, quienes, excepto en el caso de existir convenio entre los mismos, tendrán que aceptar con toda seguridad el precio de venta monopolístico." (p.237) 
mismo bien (discriminación de precios) o para bienes similares ${ }^{5}$ (diferenciación de precios). El precio que se fija en el modelo de competencia perfecta es único y equivale al costo marginal de producción; mientras que en los modelos de competencia imperfecta los precios se fijan a un nivel por encima del costo marginal de producir el bien, con el fin de obtener mayores ganancias $y$, en algunos casos, (monopolio discriminado) permitir extraer, además de la renta total del productor, toda la renta de los consumidores que participen en el mercado.

\subsection{Competencia Perfecta}

En competencia perfecta con bienes homogéneos, al consumidor le es indiferente comprarle a cualquier vendedor. Si uno de los vendedores decidiera aumentar el precio, los compradores optarian por adquirir el bien a otro que mantuviera el precio bajo, desanimando a cualquier otro vendedor a aumentar el precio. En eso consiste el principio de substitución perfecta que existe entre bienes (Katz y Rosen, 1995).

En el Modelo de Competencia Perfecta, tal y como se observa en el Gráfico 1 , las curvas de demanda $\left(D_{x}\right)$ y de ingreso marginal $\left(\mathrm{M}_{\mathrm{g}}\right)$ coinciden.

El punto de equilibrio del mercado está representado por el punto $e_{0}$ que es la intersección de la curva de demanda $\left(D_{\mathrm{x}}\right)$ con la curva de costo marginal
$\left(\mathrm{CM}_{\mathrm{g}}\right)$, que también representa a la curva de oferta del productor. El precio de equilibrio es poy la cantidad ofrecida en equilibrio es $X_{0}$. Este precio es único en el mercado debido a la homogeneidad del bien, representada en el Gráfico 1 con una curva de demanda horizontal (perfectamente elástica) y, ningún vendedor podrá manejar precios diferentes al ya fijado por el mercado.

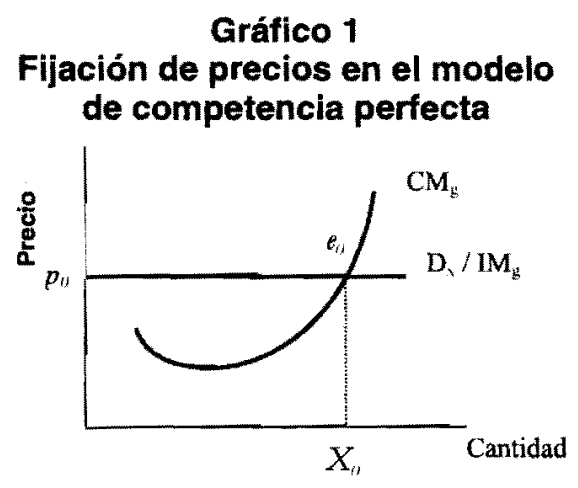

La curva de demanda perfectamente elástica, que enfrenta el vendedor en competencia perfecta, establece que el consumidor posee una sensibilidad absoluta ante cualquier cambio en el precio del bien. Esa sensibilidad absoluta significa que ante el más pequeño cambio en el precio, el consumidor no demandará unidad alguna del bien y, el vendedor no tiene poder sobre el precio por lo que deberá aceptar el precio que el mercado imponga. 


\subsection{Competencia Imperfecta}

Si el vendedor posee suficiente poder en el mercado para modificar las características de un bien homogéneo, a fin de diferenciarlo del resto de los bienes, él podrá fijar un precio diferente al precio del resto de los bienes, puesto que su bien tendrá características distintivas en el mercado. En un mercado de bienes con propiedades diferentes ${ }^{6}$, si uno de los vendedores decidiera aumentar el precio, los compradores que ya han preferido a ese vendedor continuarán comprándole a él, mientras el bien ofrecido siga adaptándose a las necesidades de los compradores y el beneficio marginal de los consumidores sea mayor que el costo marginal de producirlo. En cierta medida, debido al poder que tiene el productor para modificar las características del bien, se logra una diferenciación sobre la base de la diversidad de los productos ofrecidos, por lo tanto los usuarios deberán adaptarse a la oferta de tales productos (Andrade, 2000).

Cuando un vendedor manipula un bien para hacerlo diferente del resto de los bienes, se genera un mercado de competencia imperfecta, con bienes sustitutivos imperfectos ${ }^{7}$ (Stiglitz, 1994). Un vendedor, a través de la heterogeneidad de un producto sustitutivo imperfecto, tiene suficiente poder en el mercado para fijar precios; de alli que la curva de demanda a la que se enfrenta el vendedor no sea plana, sino inclinada con pendiente negativa (Gráfico 2). Esta inclinación de la curva de demanda representa tanto el rango de precios que puede manejar el

\section{Gráfico 2 \\ Curva de demanda en competencia monopolística}

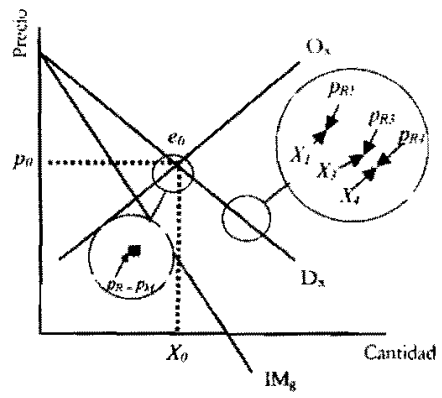

vendedor, como la heterogeneidad del bien ofrecido.

Todos los puntos de la curva de demanda $\left(D_{x}\right)$ corresponden a un precio máximo o de reserva ${ }^{B}\left(p_{R}\right)$ que estaría dispuesto a pagar cada consumidor por una determinada cantidad del bien (Katz y Rosen, 1995; Maddala y Miller, 1991)

6 Bienes heterogéneos o bienes sustitutivos imperfectos.

7 Este es el caso en el que las empresas en competencia imperfecta producen bienes lo suficientemente parecidos como para poder utilizarlos para muchos de los mismos fines (bienes que son sustitutivos imperfectos), pero lo suficientemente distintos como para que uno pueda ser algo mejor que otro. (Stiglitz, 1994: 452).

8 Rothschild (1974) define al precio de reserva como: "(..) un precio $p_{A}$ tal que el consumidor aceptará todos los precios menores o lguales a pR y rechazará todos los precios mayores que $\rho_{R} .{ }^{\prime \prime}$ (p.701), para una cantidad determinada del bien, en un momento determinado. 
Discriminación de Precios en Mercados Electrónicos

Andrade Castro, Jesús Alberto y Córdova, Evelyn

para satisfacer el deseo que tienen por obtener el producto; sin embargo, debido a la inclinación de la curva de demanda ${ }^{9}$, no todos los precios de reserva de los distintos consumidores se convertirán en el precio que el mercado fije ( $p M)$, ni se convertirán en el precio de venta $(p v)$ que fije y cobre ef vendedor monopolista. Los precios de reserva que están sobre la curva de demanda son precios máximos hipotéticos que los consumidores estarían dispuestos a pagar por una determinada cantidad del bien en un momento dado. Ellos representan los precios máximos que cada consumidor estará dispuesto a pagar si su deseo por obtener el producto es maximizado. Cualquier precio que fije el productor por debajo del precio de reserva de cada consumidor, corresponderá a la renta que ese consumidor obtendría al adquirir un bien por debajo de su precio de reserva.

El "precio de mercado" $\left(p_{M}\right)^{10}$, representado por $p_{0}$ en el Gráfico 3, se determina a través de la intersección de la curva de demanda con la curva de oferta (curva de costo marginal) en el punto eo. Este precio de equilibrio no es el precio cobrado cuando el vendedor posee poder suficiente para manipular precios. El precio que el vendedor está en capacidad de fijar y cobrar es el precio de venta pvy que en el Gráfico que se representa como $p_{1}$; siendo er el punto donde el precio de reserva coincide con el precio de venta $\left(p_{R}=p v\right)$.

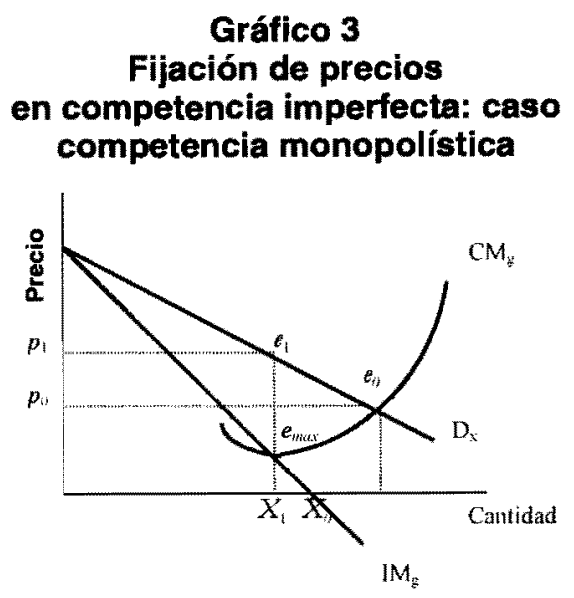

En el caso de competencia monopolística, la fijación del precio se hace a través de la regla de maximización de ganancias, lo que requiere, primero, ubicar el punto donde $1 \mathrm{M}_{\mathrm{g}}=\mathrm{CM}_{\mathrm{g}}$, que en el Gráfico 3 corresponde con el punto emax. En ese punto, el productor selecciona el nivel de producción $X_{1}$, para proyectar el nivel de precios sobre la curva de demanda, y así obtener el precio más alto que deberá fijar para maximizar su beneficio. El punto $\left(p_{1}, X_{1}\right)$ corresponde a la maximización del beneficio. Puede observarse que el precio, $p_{1}$, es mucho más alto que el precio de equilibrio que se obtendría si el vendedor no tuviese poder en el mercado (po); y en este caso, la cantidad que debería producirse del bien para maximizar las ganancias $\left(X_{1}\right)$, sería mucho menor que la cantidad necesaria para cumplir con la

9 En el caso de competencia perfecta, cualquier punto sobre la curva de demanda representa el precio de venta del mercado.

10 Económicamente el punto eo (punto que se usa para deteminar el precio de mercado o equilibrio) se entiende como el punto donde el beneficio es cero. 
condición de eficiencia (beneficio cero) en la asignación de recursos productivos $\left(X_{0}\right)$.

\section{La información como fuente de poder en los mercados electrónicos}

Uno de los fundamentos que permite sustentar al modelo de competencia perfecta como el ideal para cualquier economía, es la premisa de la información perfecta. Se supone que, bajo competencia perfecta, todos los vendedores poseen toda la información acerca de los gustos, precios de los competidores y precios de reserva de los consumidores $y$, todos los compradores poseen toda la información acerca de los bienes, precios y vendedores que están en el mercado (Stiglitz, 1994). Sin embargo, el análisis teórico señala que las asimetrías de información ocurren cuando los agentes económicos que participan en una transacción tienen diferente información acerca de la calidad del producto (Akerlof, 1970) ${ }^{11}$, que influirá en la conducta económica y en la operación del mercado antes y después de la transacción (Stiglitz, 1993) ${ }^{12}$.

Si todos los consumidores conocen todos los precios del mercado y saben dónde se encuentra el precio más bajo, entonces siempre les comprarán al ven- dedor que olrezca el precio más bajo. Como los demás vendedores no querrán perder clientes, siempre buscarán mantener el mismo precio que ofrece el competidor con precio más bajo. De esa manera se logra mantener un precio único en el mercado, gracias a la homogeneidad del bien y a la información perfecta que poseen todos los agentes. Sin embargo, muchos mercados ofrecen bienes heterogéneos y las asimetrías de información prevalecen entre los compradores y los vendedores.

En realidad, el supuesto de distribución perfecta de la información en el mercado no funciona. Becker (1982), Rothschild (1974), Stigler (1961) y Stiglitz (1994) entre otros, han señalado que la información se distribuye de una manera imperfecta y asimétrica entre los agentes que interactúan en el mercado. Por lo tanto, el conocimiento parcial (o total) que tienen los agentes acerca de las acciones que se llevan a cabo en el mercado, permite que aquellos que posean más y mejor información con respecto a sus competidores ostenten poder sobre el mercado, con consecuencias directas sobre la fijación de precios (en el caso de los vendedores) y sobre la decisión de consumo (por parte de los consumidores) ${ }^{13}$.

Diversos autores (Wigand, 1997, Schmitz, 2000, Sarkar, et al. 1995) han

11 Referida esta apreciación a los mercados de automóviles, donde el vendedor esconde información o maneja información basada en la experiencia y el conocimiento que tiene del producto.

12 Referida esta última apreciación a las fallas que se producen en los mercados de seguros.

13 Stigler (1961) expresa esta realidad en una simple pero contundente frase: "(...)la información es un recurso valioso: el conocimiento es poder." (p.213). 
estudiado la relación entre la proliferación de los mercados electrónicos y la disminución de las asimetrías de información, puesto que es de suponer que con las nuevas tecnologias de comunicación existe una tendencia a distribuir la información en forma homogénea. Si esto es así, los mercados electrónicos reducirán el poder monopolístico que ejercen los productores al ofrecer productos homogéneos en mercados cada vez más abier. tos. Sin embargo, Choi et al (1997) afirman que la brecha de las asimetrías de información es mucho más grande en el ciberespacio. $Y$ es que mucho de las asimetrias de información se producen, entre otras, por la "desconfianza" que generan tanto los productores como consumidores en los mercados. A diferencia de los mercados físicamente establecidos, donde las partes pueden ( $y$ deben) establecer un contacto físico, en el ciberespacio las partes no se reúnen, los productos no se tocan y, la confianza se resquebraja ante la posibilidad de realizar negociaciones fraudulentas. Salnoske (1997) piensa que el éxito de la economía de la internet depende de como las partes establecen confianza en sus transacciones.

\section{Discriminación de precios}

Como se ha expuesto hasta ahora, los modelos de competencia imperfecta ayudan a explicar la realidad de los mercados al mostrar que los bienes no son homogéneos y que los vendedores poseen poder para manipular el mercado. En mercados de bienes heterogéneos existe la posibilidad de discriminar precios para obtener el precio máximo que los potenciales consumidores estarían dispuestos a pagar a fin de maximizar sus deseos de obtener el bien; en este caso, el productor puede obtener el precio máximo de cada uno de los consumidores en función del precio de reserva.

Los vendedores monopolistas, asistidos por ciertos mecanismos que le ofrece el mercado, pueden obtener suficiente información de los consumidores para separarlos en grupos homogéneos o, pueden determinar cuál es la disposición máxima a pagar que los consumidores tienen, con el fin de realizar una práctica de fijación de precios conocida como discriminación de precios.

No se debe confundir diseriminación de precios con diferenciación de precios. Si bien es cierto que ambos conceptos son parecidos, existe una diferencia fundamental que hace que éstos se traten por separado. La diferencia subyace en la estructura de costos del bien objeto de discriminación o de diferenciación, según sea el caso.

Diferenclación de precios se refiere al cobro de diferentes precios a diferentes personas por diferentes unidades de un bien, como resultado de diferencias en el costo del servicio o por diferencias en el costo marginal de ese bien (Miller y Meiners, 1989). Cuando se habla de diferenciación de precios, se está hablando de diferencias en los precios de un bien, que reflejan diferencias en sus costos (Miller y Meiners, 1989; Piras, 1993).

La discriminación de precios consiste en fijar diferentes precios a diferentes consumidores por un mismo bien, por razones que no están relacionadas con diferencias en los costos de producir 
el bien (Katz y Rosen, 1995; Maddala y Miller, 1991; Piras, 1993). Cuando se habla de discriminación de precios, se refiere a dos casos: a) refiere al cobro de distintos precios unitarios por unidades sucesivas adquiridas de un mismo bien, a diferentes consumidores cuando los costos marginales para ese bien son iguales; b) refiere al cobro del mismo precio por unidades diferentes de un mismo bien (a un mismo nivel de producción y de ventas unitarias) a diferentes clientes; aún cuando los costos marginales de producción difieren para cada cliente (Maddala y Miller, 1991; Miller y Meiners, 1989).

Los bienes de información, debido a las caracteristicas propias de permitir su transferencia con el uso de tecnología electrónica, se adaptan perfectamente a los mercados que utilizan la discriminación de precios como mecanismo para obtener de los consumidores su precio de reserva. Diversos autores (Andrade, 2000; Bailey, 1998; Odlyzco, 1996; Smith et al., 1999; Varian, 1995 y 1996) citan a la discriminación de precios como el mecanismo más utilizado para fijar el precio de los bienes de información que se distribuyen a través de los mercados electrónicos, aún cuando la estructura de estos mercados no corresponda a la de un monopolio.

\section{El mercado ideal para el discriminador de precios}

Hasta los momentos se ha establecido que el mecanismo de fijación de precios comúnmente usado para los bienes de información, es la discriminación de precios. Lo importante ahora es saber por qué es asi y cuáles son las condiciones que deben estar presentes en un mercado para que éste sea considerado "ideal" para discriminar precios. Para ello, se hace necesario revisar la teoria clásica acerca de discriminación de precios, con el fin de conocer cuáles son esas condiciones ideales y qué beneficios le reportarian al vendedor llevar a cabo esa práctica en el mercado.

A.C. Pigou analizó exhaustivamente los mecanismos de la discriminación de precios bajo una perspectiva netamente económica que es independiente de los aspectos legales, morales o socioculturales que forman parte de la relación productor-consumidor. El estudio de Pigou analiza los aspectos de formación de precios basados en la satisfacción máxima de las necesidades del consumidor $y$ su deseo por abtener el bien, por lo tanto no considera a la discriminación de precios como un mecanismo ofensivo al consumidor.

Para comprender las condiciones que deben existir al considerar un mercado ideal para discriminar precios, debe partirse de la siguiente interrogante, ¿qué motiva a un vendedor a discriminar sus precios? La primera respuesta estará asociada al incentivo del productor para obtener el máximo de las ganancias extraordinarias. Sin embargo, no toda práctica discriminatoria de precios es rentable (Maddala y Miller, 1991; Pigou, 1946), ello dependerá entre otras cosas del segmento de mercado, accesibilidad, tamaño y poder de compra. De esta manera, la primera respuesta lleva a realizar una segunda interrogante, ¿qué situaciones y condiciones hacen rentable la discriminación de precios para querer ser adoptada por un vendedor? 
Lo que hace rentable o no la discriminación de precios como mecanismo válido para obtener el máximo de las ganancias extraordinarias puede encontrarse en la siguiente cita, "un mercado ideal para el productor, que le permita ofrecer sus productos y obtener la máxima ganancia de cada uno de los posibles compradores, está basado en el deseo que tienen los consumidores de obtener los productos que satisfagan mejor sus necesidades; en tal caso sería deseable establecer precios discriminatorios entre cada uno de ellos" (Andrade, 2000: 222).

Ese deseo de los consumidores por obtener los productos que satisfagan mejor sus necesidades puede ser medido económicamente utilizando el precio de resenva. Puesto que el precio de reserva expresa la cantidad máxima de dinero que estaría dispuesto a pagar cada uno de los consumidores por una determinada cantidad de un bien, ese deseo del consumidor por apropiarse de ese bien puede entonces verse reflejado en este particular precio, mientras más alto sea el precio de reserva con respecto al precio de venta, mayor será el deseo del consumidor por obtenerlo. Si el vendedor está en capacidad de conocer de antemano cuáles son los precios de reserva de los consumidores que él atiende en el mercado, entonces estará en capacidad de discriminar precios de forma rentable, debido a la diferencia que existe en cada uno de los consumidores en expresar su deseo por obtener el bien al pagar la máxima cantidad de dinero que él dispone. En este sentido, el discriminador podrá cobrar un precio más alto a quienes tengan precios de reserva altos y cobrará precios más bajos a quienes tengan precios de resenva más bajos; esto implicará que el vendedor podrá obtener todos los ingresos posibles en el mercado (si se iguala el precio de reserva con el precio de venta), pues le venderá tanto a aquellos que pueden pagar un precio único alto, como a aquellos que no podrian hacerio de tener que pagar un único precio.

La discriminación de precios será más ventajosa si ninguna de las unidades vendidas en un mercado puede transferirse a otro consumidor que se encuentre en ese mismo mercada. Si hiciera esa transferencia de un consumidor a otro que tiene precio de reserva mayor que el del consumidor que transfiere el bien (a través de la reventa, por ejemplo), entonces el vendedor correria el riesgo de perder el beneficio extra de cobrar un precio de venta más alto al consumidor a quien se le transfiere el bien, puesto que ese consumidor estaba en disposición de pagar más por el mismo bien, si se hubiese visto obligado a comprarlo directamente al vendedor.

Particularmente, la discriminación está asociada a las caracteristicas diferenciales que tiene la población consumidora. La base de la discriminación del precio está sujeta a valores exógenos al producto, puesto que tal discriminación depende del valor que cada consumidor le otorgue al producto, dependiendo del deseo de éste por obtenerlo, como el deseo de pagar el precio solicitado por el productor.

\section{Conclusiones}

1. El comercio electrónico puede ser complementario del comercio tradicional y por lo tanto el tipo de compe- 
tencia que genera no excluye la posibilidad de entrar en competencia con los bienes y servicios que se comercializan en los mercados físicos.

2. Los niveles de información del comercio físico es consecuencia de una escasez de información y los diversos niveles de información que generan las asimetrías en el comercio electrónico están basados en una sobre abundancia de información impulsada por las tecnologías de información.

3. Las diferencias en los niveles de información es lo que fundamenta el desplazamiento del modelo clásico de competencia perfecta como paradigma para el entendimiento de la formación de precios.

4. El análisis de la formación de precios está basado en la satisfacción máxima de las necesidades del consumidor y su deseo por obtener el bien.

5. El mecanismo de discriminación de precios, requiere que los productores tengan un alto nivel de información puesto que la fijación de precios está basada en el precio de reserva de los consumidores.

6. Los niveles de información del comercio electrónico generan asimetrías basadas en una sobre abundancia de información, que hace inapropiado al modelo clásico de competencia perfecta para entender los mecanismos de formación de precios en los mercados electrónicos.

7. Los bienes de información, debido a las características propias de permitir su transferencia con el uso de tecnología electrónica, se adaptan perfectamente a los mercados que utilizan la discriminación de precios como mecanismo para obtener de los consumidores su precio de reserva.

\section{Bibliografía citada}

Akerlof, G.A.(1970), "The Market for "Lemons": Quality Uncertainty and the Market Mechanism', Quarterly Journal of Economics, Vol.84, August, pp.488500.

Andrade, Jesús A. (2000). "Formación de precios de los productos de información en mercados que funcionan en redes". Revista Venezolana de Gerencia, Año 5 № 11, Venezuela, La Universidad del Zulia, Vice - Rectorado Académico, pp. 209-228.

Bailey, J. (1998). Intermediation and Electronic Markets: Aggregation and Pricing in Internet Commerce, Ph.D. Thesis, Technology, Management and Policy, Massachusetts Institute of Technology, May, Massacusetts, EEUU.

Becker, G. (1982). Teoría Económica. 1" reimpresión; Fondo de Cultura Económica, México.

Choi, S. Y, Stahl, D. O. and Whinston, A. (1997) The Economics of Electronic Commerce, Macmillan Technical Publishing, Inidiana.

Hotelling, G. (1929). "Stability in Competition", Journal of Political Economy, 44, 423-429

Katz, M. y Rosen, H. (1995). Microeconomía. Primera edición en español, Irwin, España.

Maddala, G. y Miller, E. (1991). Microeconomía. Primera edición en español, Edit. McGraw-Hill, México.

Miller, R. y Meiners, A. (1989). Microeconomía. Segunda edición en español, Edit. McGraw-Hill, Bogotá, Colombia.

Odlyzco, A.(1996). "The Bumpy Road of Electronic Commerce". Tomado de: Mau- 
rer, H. (editor). WebNet96 World Conference Web Society Procceedings, pp. 378-389.

Pigou, A. (1946). La Economia del Bienestar, Editorial Aguilar, Madrid, España

Piras, C.(1993). Aspectos Económicos de la Política de Competencia. Superintendencia para la Promoción y Protección de la Libre Competencia, Ministerio de Industria y Comercio, Venezuela. Consulta más reciente: 25/09/00 Disponible: http://www.procompetencia.gov.ve/aspectoseconomicos.html.

Robinson, J. (1934). "What is imperfect Competition?" The Quaterly Journal of Economics, November, pp. 104-120.

Rothschild, M (1974). "Searching for the Lowest Price When the Distribution of Prices is Unknown". Journal of Political Economy, Volume 82, Issue 4, August, pp. 689-711.

Salnoske, K. (1997) "Buidling Trust in Electronic Commerce" Credit World, Jul/Aug.

Sarkar, M. B., Butler B., \& Steinfield, C. (1995). "Intermediaries and cybermediaries: A continuing role for mediating players in the electronic marketplace". Journal of Computer-Mediated Communication, 1 (3) Consulta más reciente: 20/12/2000.

Disponible: http://www.ascusc.org/jcmc/vol1/ issue3/vol1no3.htmi
Schmitz, Stefan (2000). "The Effects of Electronic Commerce on the Structure of Intermediation". Journal of Computer-Mediated Communication (JCMC) 5 (3) March.

Smith, M; Bailey, J. y Brynjolfsson, E. (1999). Understanding Digital Markets: Review and assesment. MIT Sloan School of Management Working Papers. Consulta más reciente: 15/12/99 Disponible: http:/iecommerce.mit.edu/papers/ude

Stigler, G. (1961). "The Economics of Information." Journal of Political Economy. Volume 69, Issue 3, June, pp. 213 225.

Stiglitz, J.E.(1993), Economics, W.W. Norton \& Company, New York, USA.

Stiglitz, J.E. (1994). Principios de microeconomia. $1^{a}$ edición, Editorial Ariel, España.

Varian, $H(1995)$. Pricing Information Goods. Working paper. Department of Econo. mics, University of Michigan, Ann Arbor, Michigan, EEUU.

Varian, H. (1996) "Differential Pricing and Efficiency". Firstmonday, issue 2, August. Consulta más reciente: 29-062000. Disponible: http:/firstmonday.org/issues/issue2/different/index.ntml

Wigand, R. T. (1997). "Electronic commerce: definition, theory, and contex". The Information Society, $13(1), 1-16$. 
rer, H. (editor). WebNet96 World Conterence Web Society Procceedings, pp. 378-389.

Pigou, A. (1946) . La Economía del Bienestar, Editorial Aguilar, Madrid, España

Piras, C.(1993). Aspectos Económicos de la Política de Competencia. Superintendencia para la Promoción y Protección de la Libre Competencia, Ministerio de Industria y Comercio, Venezuela. Consulta más reciente: $25 / 09 / 00$ Disponible: http://www.procompetencia.gov.velaspectoseconomicos.html.

Robinson, J. (1934). "What is Imperfect Competition?" The Quaterly Journal of Economics, November, pp. 104-120.

Rothschild, M (1974). "Searching for the Lowest Price When the Distribution of Prices is Unknown". Journal of Polltical Economy, Volume 82, Issue 4, August, pp. 689-711.

Salnoske, K. (1997) "Buidling Trust in Electronic Commerce" Credit World, JullAug.

Sarkar, M. B., Butler B., \& Steinfield, C. (1995). "Intermediaries and cybermediaries: A continuing role for mediating players in the electronic marketplace". Journal of Computer-Mediated Communication, 1(3) Consulta más reciente: 20/12/2000.

Disponible: http:/www.ascusc.org/jemc/vol1/ issue $3 /$ vol $1 \mathrm{no} 3 \mathrm{html}$
Schmitz, Stefan (2000). "The Effects of Electronic Commerce on the Structure of Intermediation". Journal of Computer-Mediated Communication (JCMC) 5 (3) March.

Smith, $M_{;}$Bailey, J. y Brynjolfsson, E. (1999). Understanding Digital Markets: Review and assesment. MIT Sloan School of Management Working Papers. Consulta más reciente: $15 / 12 / 99$ Disponible: http://ecommerce.mit.edu/papers/ude

Stigler, G. (1961). "The Economics of Information." Journal of Political Economy. Volume 69, Issue 3, June, pp. 213225.

Stiglitz, J.E.(1993), Economics, W.W. Norton \& Company, New York, USA.

Stiglitz, J.E. (1994). Principios de microeco-

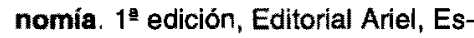
paña.

Varian, H (1995). Pricing Information Goods. Working paper. Department of Economics, University of Michigan, Ann Arbor, Michigan, EEUU.

Varian, H. (1996) "Differential Pricing and Efficiency". Firstmonday, issue 2, August. Consulta más reciente: 29-062000. Disponible: http:/firstmonday.org/issues/issue2/differentindex.html

Wigand, R. T. (1997). "Electronic commerce: definition, theory, and contex". The Information Society, 13 (1), 1-16. 